\title{
1 Dietary restriction and lifespan: adaptive reallocation or somatic
} sacrifice?

\author{
Matthew DW Piper *, Brooke Zanco *, Carla M Sgrò *, Margo I Adler ${ }^{\S}$, Christen K Mirth *, Russell \\ Bonduriansky ${ }^{\dagger}$
}

\author{
* School of Biological Sciences, Monash University, Australia \\ $\S$ Beaker St, Tasmania, Australia \\ † School of Biological, Earth and Environmental Sciences, University of New South Wales, \\ Australia
}

\begin{abstract}
:
Reducing overall food intake or lowering the proportion of protein relative to other macronutrients, can extend lifespan in diverse organisms. A number of mechanistic theories have been developed to explain this phenomenon, mostly assuming that the molecules connecting diet to lifespan are evolutionarily conserved. A recent study using Drosophila melanogaster females has pinpointed a single essential micronutrient that can explain how lifespan is changed by dietary restriction. Here, we propose a likely mechanism for this observation, which involves a trade-off between lifespan and reproduction, but in a manner that is conditional on the dietary supply of an essential micronutrient - a sterol. Importantly, these observations argue against previous evolutionary theories that rely on constitutive resource reallocation or damage directly inflicted by reproduction. Instead, they are compatible with a model in which the inverse relationship between lifespan and food level is caused by the consumer suffering from varying degrees of malnutrition when maintained on lab food. The data also indicate that animals on different lab foods may suffer from different nutritional imbalances and that the mechanisms by which dietary restriction benefits the lifespan of different species may vary. This means that translating the mechanistic findings from lab animals to humans will not be simple and should be interpreted in light of the range of challenges that have shaped each organism's lifespan in the wild and the composition of the natural diets they would feed on.
\end{abstract}

\section{Introduction:}

Ageing can be defined as the progressive loss of organismal function that ultimately leads to death [1]. Organisms in the wild are thought to be less prone to the fitness costs of ageing than do organisms in the lab because the lives of wild organisms tend to be cut short by external hazards, such as infection or predation [2]. Because of this, each organism's schedule of reproduction must be tuned to its environment to maximise reproductive success before they are likely to die [3-5]. But reproduction requires a heavy investment of time and nutritional resources - both of which could be spent in other ways, such as fighting off infection or repairing cellular damage to maintain somatic integrity $[6,7]$. Understanding the mechanistic basis of how these investments are balanced is thought to be centrally important for understanding the processes that cause ageing [8], and thus provide insights into how we might intervene to improve health and extend lifespan. 
49 The availability of food, and its nutritional components, is critical in determining how organisms make 50 investments in reproduction and lifelong health [9]. By manipulating nutrition, experimental biologists have 51 observed that dietary restriction (DR) without malnutrition can extend lifespan in a broad range of taxa, but that doing so often results in lowered reproduction [10-12]. In attempts to dissect this phenomenon further, an enormous effort has gone into manipulating the severity, timing and quality of restriction that must occur

\section{4} 55 for the animals to be longer lived [13]. Amongst the various beneficial interventions, manipulating the dietary macronutrient content to lower the ratio of protein : non-protein derived energy (from carbohydrate + fat), without restricting intake, can promote longevity [14].

In parallel with these discoveries, research has identified that major nutrient sensing pathways, such as Target of Rapamycin (TOR) and insulin and insulin-like growth factor (IGF)-like signalling (IIS), are crucial determinants of lifespan $[15,16]$. When nutrients are abundant, these signalling pathways promote growth, reproduction, and nutrient storage. However, when nutrient levels drop, the signals promote protective processes, while also inducing the turnover of stored macromolecules to supply the building blocks and biochemical reducing power that is required to protect cellular function [17]. It is thought that by turning down growth signalling and by recycling old cellular material, the organism expresses a leaner and better functioning proteome that is key to enhanced lifespan [18]. In particular, the TOR kinase is an evolutionarily conserved amino acid sensor and signalling molecule that has attracted a great deal of attention in ageing research, since its suppression by genetic or pharmacological means can extend lifespan in a range of organisms [17]. When considered together with the pro-longevity effects of protein restriction, these data suggest that lowered dietary protein acts via reduced TOR signalling to extend life.

In recent work on the fruitfly Drosophila melanogaster, Zanco et al. have expanded understanding of these events, by establishing that an essential dietary micronutrient, a sterol, is the key determinant of lifespan in response to DR when the dietary protein : carbohydrate ratio is experimentally reduced [19]. These data offer a new explanation for how lowered dietary protein and lowered TOR signalling prolong life in a way that does not appear to require phenotypic trade-offs or enhanced damage repair. Instead lifespan is preserved on lower protein diets because the flies do not suffer from a nutrient imbalance as a result of sterol deficiency, and so they avoid somatic damage. These data suggest a need to rethink the mechanistic basis of DR in Drosophila, and to critically evaluate how broadly the data from Drosophila studies can inform our understanding of evolutionarily conserved mechanisms of lifespan determination and ageing.

\section{How does DR extend lifespan?}

Probably the most prominent theory to explain the mechanisms by which DR extends life is built upon the Disposable Soma theory of ageing [20-23]. This theory proposes that organisms monitor the availability of limiting nutritional resources (often referred to as "energy") and then strategically allocate them to biochemical processes that alter phenotypes to maximise fitness. According to this "resource reallocation" hypothesis, when food is scarce, energy is invested away from reproduction and into maintaining the soma. This strategy is assumed to enhance fitness by increasing the probability of surviving to a point where food becomes available and conditions for offspring survival improve, at which point reproduction can resume. By 
contrast, when resources are abundant, energy is prioritised for reproduction and redirected away from somatic maintenance, so that high reproductive effort coincides with a time when reproduction is most likely to be successful. Importantly, it is assumed that the degree of investment into somatic maintenance regulates ageing, causing animals under DR to live longer - a benefit that is traded for reduced reproduction $[23,24]$. While this explanation for DR's effects on longevity and reproduction is intuitively appealing, it is challenged by new data. In particular, two key elements of the theory are now in question: the potential for nutrient reallocation from reproduction to somatic maintenance to enhance fitness, and the nature of the lifeshortening damage that brings early death when feeding on high nutrient diets.

Adler and Bonduriansky considered the resource reallocation hypothesis in the context of natural populations experiencing high extrinsic (background) mortality and physiological stress [25]. They pointed out that for many organisms, including small-bodied animals such as insects, postponing reproduction is unlikely to enhance fitness because the risk of mortality from extrinsic causes, such as predation, is very high. Moreover, because DR tends to reduce animals' ability to cope with some of the key challenges posed by natural environments - such as the need to thermoregulate, mount an immune response to infection, and heal wounds - animals subject to DR are likely to have especially poor survival prospects in the wild. This suggests that, to maximise fitness, most animals should make the most of the limited nutrients they have available by investing as heavily as possible into immediate reproduction. As part of this strategy, any nutrients that are limiting may need to be supplemented from somatic stores. In the process of retrieving these stores, organisms reduce their own biomass, which reduces the burden of somatic maintenance, while at the same time also recycling old and dysfunctional cellular components - a process that is thought to have beneficial anti-ageing effects and generally prolongs life in the benign, protected conditions of the laboratory [25]. This type of inadvertent benefit of nutrient recycling is different from the strategic resource reallocation of the Disposable Soma theory, which is assumed to enhance fitness by making the animal more robust $[20,22,23]$. Instead, depleting stored biomass to maximise reproduction might enable DR animals to achieve some reproductive success despite nutrient limitation, but it will also make them frail and sensitive to a range of environmental stressors $[20,23,25]$. Thus any lifespan benefit of DR observed for animals such as Drosophila melanogaster might simply be an artefact of the protected lab environment, where almost all extrinsic hazards are removed.

The second important challenge to the resource reallocation explanation for DR is evidence that raises new questions about the nature of the physiological process that modifies lifespan $[19,26,27]$. This is important because this type of data informs our understanding of the mechanisms by which DR extends life. In Holliday's original formulation, turnover of dysfunctional proteins and/or protection of macromolecules from reactive oxygen species were cited as probably being important for somatic maintenance under DR [23]. This type of damage, especially to the proteome, is still considered to be an important determinant of lifespan, which is why upregulation of autophagy in response to reduced TOR signalling is thought to be protective $[18,25,28,29]$. It is also possible that high food and reproduction compromise the soma in a way that makes resource reallocation for repair irrelevant. For instance, lifespan could be shortened by physical damage from reproduction [27] or the inappropriate continuation of developmental programs into older age [26]. In either scenario, lifespan would still be inversely related to nutrient supply, and the nutritional conditions that promote growth and reproduction would always shorten lifespan. 
133 But data from Drosophila argues against this point since reproduction and lifespan can be maximised simultaneously if the dietary amino acid composition is manipulated to match the amino acid profile of the consumer's exome [30,31]. This new data challenges the ideas of the Disposable Soma theory and suggests two alternative possibilities for the lifespan-extending effect of DR. One possibility is that high levels of nutrients lead to greater nutrient signalling, which in itself can shorten lifespan independently of its function in reproduction [25,32]. Another possibility is that the diets we use for experiments are nutritionally imbalanced in such a way that the observed inverse relationship between lifespan and food level is a reflection of the consumer suffering from varying degrees of malnutrition [25]. This is of particular concern for labs working with Drosophila since the lack of diet standardisation means it is possible that each lab is subjecting their flies to a different type of nutrient imbalance that affects lifespan in a different way [33-36]. If true, then mechanistic studies on DR in flies may not yield useful information about how diet affects lifelong health in other organisms.

\section{Sterol limitation mediates a conditional lifespan / reproduction trade-off in Drosophila}

During the last 15 years, many studies across several organisms have found that lifespan and reproduction are optimised by diets with a different balance of macronutrients $[9,37,38]$. In particular higher protein : carbohydrate ratios are associated with greater reproduction and shorter lifespan, while lower protein : carbohydrate diets are associated with lower reproduction and longer lifespan. In recent work on Drosophila, Zanco et al. have extended these findings by showing that adding sterols to the diet of flies on high protein : carbohydrate diets can extend their lifespan without compromising reproduction [19]. Thus, both traits share the same nutritional optimum. This shows that neither high reproduction, nor high dietary protein, nor high levels of nutrient-related signalling are directly causal in shortening fly lifespan. Instead, when dietary sterols are low, fly lifespan is traded off against reproduction, but when dietary sterols are sufficient, the two traits are determined independently of one another. In other words, flies on lab food suffer from a lifespan limiting nutrient imbalance (sterol shortage) that worsens as increasing food concentrations promote higher levels of reproduction. This observation supports the idea that Drosophila studies of "DR" may reveal more about how different intensities of nutrient imbalance vary fly lifespan, rather than advancing our understanding about how an evolutionarily conserved mechanism of DR can extend lifespan [25].

\section{How might sterol limitation modify lifespan in Drosophila?}

It appears that although many nutrients are required to form an egg in Drosophila, flies only sense some of those nutrients when committing to egg production [39,40]. Specifically, when dietary protein levels are high and there are adequate levels of dietary carbohydrates and metal ions, egg production will be initiated, even when other essential components, such as sterols, are undersupplied [40]. In order to make up for this sterol deficit, the flies source them from somatic tissue and deliver them to the ovary to ensure the production of high quality eggs $[19,41,42]$. In doing so, the flies deplete their own sterols, which shortens their lifespan - a strategy that might enhance fitness in natural populations by enabling flies to take advantage of valuable reproductive opportunities. However, when dietary protein is low, only small quantities of sterols are required 
173 for reproduction and so the mother lives longer by avoiding the costs of sterol depletion. Not only is this model supported by the fact that adding sterols to the food of egg laying females on high protein : carbohydrate diets causes them to be long lived, but also by the fact that sterol deficit diets do not lead to reduced lifespan in genetically sterile females $[19,40]$.

One of the ways in which sterol depletion may shorten fly lifespan is by compromising the integrity of the Drosophila gut, which has already been recognised as a key determinant of lifespan in numerous papers [43-49]. Specifically, gut barrier function declines $\sim 48 \mathrm{~h}$ prior to death and this can be associated with susceptibility to DR $[46,48]$. In Drosophila, the fat body, which functions in a similar manner to mammalian adipose tissue, serves as a major nutrient reservoir [50]. Upon dietary sterol deprivation, a fat-body specific sterol esterase, $\mathrm{Hsl}$ (Hormone sensitive lipase), is activated and the retrieved sterols are transported to the ovaries for egg production [41]. In the absence of $\mathrm{Hsl}$, a gut specific sterol esterase, called Magro, is upregulated [41]. These data may indicate that there is a program of sterol mobilisation that is initiated to meet the needs for reproduction. This starts with the fat body, then draws on reserves in other tissues. If activation of the gut-specific sterol esterase indicates that sterols are drawn from the gut, then eventual depletion of these stores could lead to reduction of free sterols and cellular membrane integrity, which leads to loss of barrier function that compromises lifespan. If this is the case, then mutating the gut sterol esterase Magro should preserve gut function and cause the flies to be longer lived on sterol depleted food. In addition, we predict that once fat body sterol reserves are depleted, Magro mutant flies might lay sterol depleted eggs that are likely to be non-viable.

A closely related, but slightly different mechanism, is supported by data from several recent papers demonstrating a role for ecdysteroid signalling from the ovary to the gut to stimulate female gut growth in response to mating $[44,51,52]$. Ecdysone-induced gut growth is thought to enhance nutrient absorption to facilitate higher levels of reproduction. If gut growth is also stimulated by higher protein : carbohydrate diets $[53,54]$, then flies on low sterol diets may be at risk of trying to build gut tissue without sufficient resources, which could compromise barrier function in a way that leads to earlier death. If this is the case, inhibiting gut growth, by gut-specific mutation of the ecdysone receptor $(E c R)$, should protect gut function and extend life on low sterol foods. Indeed, gut specific knockdown of EcR has already been shown to suppress agedependent gut dysplasia [44] and lowering growth-related TOR signalling in the gut can extend lifespan $[55,56]$. Because smaller guts would reduce nutrient absorption, we would also predict that these interventions would reduce the ability to increase egg production on high nutrient diets.

We also predict that death in either of these scenarios would be preceded by loss of gut integrity, unless the diet was supplemented with sterols, in which case the gut and lifespan should be preserved. Finally, sterols have been implicated as important for cold acclimation in Drosophila [57] indicating that sterol limited flies under DR may take less time to succumb to a chill coma or have worse survival prospects following cold conditions. If true, these experiments would pinpoint the cause of diet-mediated fly death on higher protein : carbohydrate diets.

\section{Does dietary sterol limitation form the mechanistic basis of DR?}


Here, we propose that flies match their egg production to the level of dietary protein : carbohydrate irrespective of the availability of dietary sterols, which are essential for the mother's survival. Thus, the nutrients that inform the decision to commit to reproduction are different from the nutrient that is traded between lifespan and reproduction when dietary intake is limited. This is an important difference from past theories of DR in which the limiting nutrient is both the molecule being sensed and the resource that is being traded between lifespan and reproduction. When the two are coupled, investment decisions are informed by the actual availability of the critical resource, but when the two are separated, flies are much more likely to make the decision to invest in reproduction despite a potential life-threatening shortage of other resources that they also need. The extent to which this potential problem actually poses a threat to evolutionary fitness depends on how sterols actually vary in the flies' natural diet and if they do, to what extent the flies have evolved behavioural and/or physiological strategies with which to buffer against dietary sterol deficiencies.

\section{Do sterol levels in fly food vary?}

$D R$ is studied in the lab while maintaining flies on artificial diets that are assumed to approximate their natural diets, which include sugar from fruit and other nutrients from the microbes, particularly yeasts, that ferment the fruit [58]. In practice, there are almost as many different recipes as there are labs, and while almost all diets include yeast as a core component, even yeast can vary in its nutritional profile as a function of the strain used and the method by which it was produced [34]. Interestingly, one of the biggest composition variations that occurs in yeast is the relative proportion of sterols it contains. Yeasts can synthesise their own sterols via a biosynthetic pathway that requires oxygen and so when oxygen availability fluctuates, the content of sterols relative to protein varies. This can range more than 10 -fold, being high under aerobic culture conditions (used in the production of baker's yeast) and low under anaerobic conditions (used during alcoholic fermentation) [59-61]. Even lower levels of sterols are expected in fly diets made from water soluble yeast extracts because their production method excludes non-polar molecules. Our past data comparing the effects of various yeast foods show that differences in production methods can account for large differences in the fecundity and lifespan of flies maintained on them, and that these differing effects can be dramatically reduced by the addition of sterols $[19,33]$. Additional tests of the degree of sterol limitation across more lab diets is required, but these data suggest that flies on popular lab foods do tend to be sterol limited.

In the fly's natural setting, yeasts growing in sessile colonies on fruit will be a mix of both aerobic, sterol producing cells on the exterior of the colony, and anaerobic sterol non-producing cells, on the interior [62]. Indeed, the fermentation products that are indicative of anaerobic microbial growth (ie alcohols and acids) are prominent features of rotting fruit to which Drosophila melanogaster is strongly attracted, indicating they are highly relevant to the flies' ecology [63]. We predict, therefore, that not only do lab diets vary in their sterol contents, but that flies have evolved in environments where dietary sterols vary. However, the degree to which wild flies are sterol limited for reproduction is yet to be determined. 
254 In order to buffer against nutritional variations, animals can vary their feeding behaviour and/or alter their

255

256

257

258

259

260

261

262

263

264

265

266

267

268

269

270

271

272

273

274

275

276

277

278

279

280

281

282

283

284

285

286

287

288

289

290

291

292

293

294

physiology $[9,64]$. Although flies exhibit strong changes in feeding preference to compensate for prior macronutrient deficiencies, direct tests show that flies do not seek out sterol-containing food after having been deprived of sterols for 3 days [65]. While there is little evidence that insects can taste sterols, they can use associative learning to find or avoid food containing particular types of sterols [66]. Thus, selective sterol feeding in Drosophila may not have been detected yet because of experimental design issues, such as the duration of deprivation and the types of foods employed. It is also possible that flies have no need for behavioural compensation because the lifespan shortening effects of sterol depletion are not sufficient to impose a real fitness cost in the wild. Interestingly, Drosophila larvae can survive low sterol foods by altering their speed of development, final body size attained, and the relative distribution of sterols in their bodies [67], while wild-caught adult flies exhibit differences in both the quantities and qualities of sterols across various body tissues [57]. Thus, flies have evolved to be flexible with their sterol usage, in particular to manage their distribution around the body. It is thus conceivable that in order to protect fecundity, flies can retrieve sterols from their own soma to produce high quality eggs in such a way that the adults can still survive to the point that extrinsic hazard would have normally killed them. Our data shows that this is possible for about 10 days from adult emergence, which is comparable to estimates of life expectancy in wild Drosophila melanogaster and other flies [68-70], suggesting that flies might be able to buffer against dietary sterol shortages in order to take advantage of dietary macronutrient levels that accommodate high egg production [42].

\section{What can we learn about DR from studies on Drosophila?}

\section{Dietary variations - implications for comparing Drosophila DR studies between labs}

We have outlined evidence that the proportion of sterols : macronutrients can determine length of life for Drosophila under DR, and that diets can vary significantly in their nutritional makeup between labs. This introduces the possibility that not all studies on lifespan responses to DR have the same mechanistic basis. Indeed, evidence for this already exists since water supplementation can account for the lifespan effects of DR in some situations [71,72], but not others [72,73]. An additional complication for interlaboratory comparisons of DR can come from differences in the range of dietary nutrient concentrations used. Our studies were originally designed using a range of food concentrations, or protein : carbohydrate ratios, in which lifespan and reproduction were inversely correlated, because on these diets flies show evidence that they have evolved strategies to maximise fitness at both high and low nutritional extremes [33,74]. However, food concentrations do exist beyond this range and these generally give rise to detrimental effects on both lifespan and fecundity (e.g. see extreme diets in [72,75-77]). Given their negative impact on the consumer, these extremely high nutrient doses appear to be toxic to the flies, which means that any benefits to lifespan seen from diluting them is mediated by avoiding toxicity. Thus the mechanisms behind lifespan change in response to extreme DR conditions are likely to be different to those at play for the moderate DR that we employ. These data point to an important consideration that when studying the mechanisms of DR, the details of the food quantity and quality matter. To gain perspective on these issues it can be useful to employ a nutritional framework, such as nutritional geometry $[9,78]$, to capture the extent of these differences. 
Dietary variations - implications for comparing DR studies between species

One of the key reasons for using model organisms in ageing research is based on the assumption that the mechanisms of ageing, and lifespan responses to diet, are evolutionarily conserved. When considering the findings of Zanco et al. (2021) in light of the fact that insects are sterol heterotrophs and mammals are sterol autotrophs, it seems unlikely that nutrient level sterol limitation is going to compromise consumer lifespan in mammals as it does in flies. However, these data point to an important, and potentially general, principle: when organisms commit to reproduction based on a limited subset of nutrients they are at risk of suffering from critical nutrient shortages that can threaten lifespan, particularly with respect to the micronutrients.

Indeed, there is evidence that a variety of organisms experience simultaneous macronutrient and micronutrient limitations that restrict growth, including specific examples of limits for protein and sterols or other essential lipids [79-82]. Rodents may also show signs of nutritional co-limitation during reproduction wherein mothers remobilise calcium from their bones and teeth to supplement infant nutrition during lactation - an effect that is exacerbated if calcium is diluted in the animals' diet [83,84]. Interestingly, when performing mouse DR studies it is routine to fortify the micronutrient supply of the cohorts with restricted food intake because of concerns about malnutrition from having less food [12]. But, if the macronutrients can drive overinvestment of micronutrients into reproduction in mice as they do in flies, this strategy may in fact be the opposite of what the animals need; fortifying the micronutrient supply of animals on high food diets may protect them from a cost of reproduction, preserve lifespan, and so eliminate at least some of the effects of diet variation on lifespan.

We also anticipate that, similar to flies, the range of diet qualities and methods for imposing DR in rodents could mean that different mechanisms operate in different situations. Indeed, the mechanistic basis of altered lifespan in response to food restriction or intermittent fasting (also called caloric restriction) may well differ from the mechanisms behind lifespan responses to altered dietary nutrient balance $[38,85,86]$. If this is true for lab studies on model organisms, then there is little doubt that these issues will be highly relevant in humans. For instance, restricting food intake of individuals in countries where nutrient consumption is likely to exceed requirements for reproduction may avoid some lifespan shortening pathologies associated with overeating (e.g. reduction in type II diabetes) but not modify the intrinsic rate of ageing. Thus, our data indicate that while DR can continue to provide useful insights into the ways that diet imbalances might modify lifespan, it is likely that DR studies across labs and species do not share a single unified mechanism that informs us about the processes that cause ageing.

It is also clear that a better understanding of the nutritional ecology of natural populations could help to unravel the physiological mechanisms mediating the effects of dietary nutrients on lifespan, and to understand the evolution of these effects. Fitness and its major components - survival and reproduction are exquisitely sensitive to environmental context, including sources of stress such as temperature fluctuations or pathogens and extrinsic mortality risks that determine life expectancy. The fitness effects of a strategy such as reallocating resources from reproduction to survival, or drawing on somatic stores of sterols 
336 for egg production, will depend in large part on whether wild flies are usually limited to a single reproductive

337 bout, or have a realistic chance of surviving long enough to take advantage of future reproductive

338 opportunities.

339

340 To answer this question for Drosophila melanogaster, we need to estimate the mortality rates experienced by

341 flies in the wild, and to understand how mortality risk is modulated by different physiological and behavioural

342 responses. Obtaining data on lifespan and reproduction in natural populations of $D$. melanogaster presents

343 many practical challenges, but techniques such as mark-recapture have been used successfully with other

344 small insects and could be adapted for research on D. melanogaster $[87,88]$. Such research could answer

345 basic questions such as whether dietary restriction extends life in natural populations, or whether lifespan

346 extension on DR is only observed under benign laboratory conditions. Insight could also be gained through

347 laboratory experiments that manipulate the quality and quantity of dietary nutrients under varying levels of

348 stress (e.g. high temperature or pathogen exposure). A better understanding of the macro- and micro-

349 nutrient content of foods available to model organisms such as D. melanogaster in the wild, as well as how

350 nutrient content and abundance vary spatially and temporally in natural environments, will also inform

351 interpretation of dietary restriction experiments in the laboratory. Extending research on diet and longevity to

352 organisms living in natural and semi-natural environments could provide valuable context for interpreting

353 results of laboratory experiments.

355 Acknowledgements

356 Many thanks to lab members who have been involved in discussions on this topic; in particular, thanks to

357 Josh Johnstone and Tahlia Fulton for reading through and commenting on the manuscript. 


\section{References}

1 López-Otín C, Blasco MA, Partridge L, Serrano M \& Kroemer G (2013) The Hallmarks of Aging. Cell 153, 1194-217.

2 Medawar P (1952) An unsolved problem of biology: An Inaugural Lecture Delivered at University College, London, 6 December, 1951. HK Lewis and Company.

3 Schaffer WM (1974) Selection for Optimal Life Histories: The Effects of Age Structure. Ecology 55, 291-303.

4 Hamilton WD (1966) The moulding of senescence by natural selection. $J$ Theor Biol 12, 12-45. 5 Law R (1979) Optimal Life Histories Under Age-Specific Predation. Am Nat 114, 399-417. 6 Partridge L \& Sibly R (1991) Constraints in the evolution of life histories. Philosophical Transactions Royal Soc Lond Ser B Biological Sci 332, 3-13.

7 Parker GA \& Smith JM (1990) Optimality theory in evolutionary biology. Nature 348, 27-33.

8 Barnes Al \& Partridge L (2003) Costing reproduction. Animal Behaviour 66, 199-204.

9 Simpson SJ \& Raubenheimer D (2012) The Nature of Nutrition: A Unifying Framework from Animal Adaptation to Human Obesity Princeton University Press, Princeton, New Jersey. 10 Fisher RA (1930) The Genetical Theory of Natural Selection. Clarendon Press.

11 Williams GC (1966) Natural Selection, the Costs of Reproduction, and a Refinement of Lack's Principle. Am Nat 100, 687-690.

12 Weindruch R \& Walford RL (1988) Retardation of aging and disease by dietary restriction. CC

Thomas.

13 Wilson KA, Chamoli M, Hilsabeck TA, Pandey M, Bansal S, Chawla G \& Kapahi P (2021)

Evaluating the beneficial effects of dietary restrictions: A framework for precision nutrigeroscience. Cell Metab.

14 Couteur DG \& Simpson SJ (2018) 90th Anniversary Commentary: Caloric Restriction Effects on Aging. The Journal of nutrition 148, 1656-1659.

15 Bjedov I \& Rallis C (2020) The Target of Rapamycin Signalling Pathway in Ageing and Lifespan Regulation. Genes-basel 11, 1043.

16 Partridge L, Fuentealba M \& Kennedy BK (2020) The quest to slow ageing through drug discovery. Nat Rev Drug Discov, 1-20.

17 Saxton RA \& Sabatini DM (2017) mTOR Signaling in Growth, Metabolism, and Disease. Cell 168, 960-976.

18 Sala AJ, Bott LC \& Morimoto RI (2017) Shaping proteostasis at the cellular, tissue, and organismal level. J Cell Biol.

19 Zanco B, Mirth CK, Sgrò CM \& Piper MD (2021) A dietary sterol trade-off determines lifespan responses to dietary restriction in Drosophila melanogaster females. Elife 10, e62335.

20 Kirkwood TBL \& Holliday R (1979) The evolution of ageing and longevity. Proc Royal Soc Lond Ser B Biological Sci 205, 531-546.

21 Kirkwood TBL (1977) Evolution of ageing. Nature 270, 301-304.

22 Kirkwood TBL \& Rose MR (1991) Evolution of senescence: late survival sacrificed for reproduction. Philosophical Transactions Royal Soc Lond Ser B Biological Sci 332, 15-24. 23 Holliday R (1989) Food, reproduction and Longevity: Is the extended lifespan of calorierestricted animals an evolutionary adaptation? Bioessays 10, 125-127.

24 Shanley DP \& Kirkwood TBL (2000) CALORIE RESTRICTION AND AGING: A LIFE-HISTORY ANALYSIS. Evolution 54, 740-750.

25 Adler MI \& Bonduriansky R (2014) Why do the well-fed appear to die young? Bioessays 36, 439-450.

26 Blagosklonny MV (2006) Aging and immortality: quasi-programmed senescence and its pharmacologic inhibition. Cell cycle (Georgetown, Tex) 5, 2087-102.

27 Tatar M \& Carey JR (1995) Nutrition Mediates Reproductive Trade-Offs with Age-Specific Mortality in the Beetle Callosobruchus Maculatus. Ecology 76, 2066-2073.

28 Speakman J (2020) Why does caloric restriction increase life and healthspan? The 'clean cupboards' hypothesis. Natl Sci Rev.

29 Matai L, Sarkar GC, Chamoli M, Malik Y, Kumar SS, Rautela U, Jana NR, Chakraborty K \& Mukhopadhyay A (2019) Dietary restriction improves proteostasis and increases life span through endoplasmic reticulum hormesis. Proc National Acad Sci 116, 17383-17392.

30 Grandison RC, Piper MDW \& Partridge L (2009) Amino-acid imbalance explains extension of 
415 lifespan by dietary restriction in Drosophila. Nature 462, 1061-4.

416

417

418

419

420

421

422

423

424

425

426

427

428

429

430

431

432

433

434

435

436

437

438

439

440

441

442

443

444

445

446

447

448

449

450

451

452

453

454

455

456

457

458

459

460

461

462

463

464

465

466

467

468

469

470

471

31 Piper MDW, Soultoukis GA, Blanc E, Mesaros A, Herbert SL, Juricic P, He X, Atanassov I, Salmonowicz H, Yang M, Simpson SJ, Ribeiro C \& Partridge L (2017) Matching Dietary Amino Acid Balance to the In Silico-Translated Exome Optimizes Growth and Reproduction without Cost to Lifespan. Cell Metab 25, 610-621.

32 Libert S, Zwiener J, Chu X, Vanvoorhies W, Roman G \& Pletcher SD (2007) Regulation of Drosophila life span by olfaction and food-derived odors. Science (New York, NY) 315, 1133-7. 33 Bass TM, Grandison RC, Wong R, Martinez P, Partridge L \& Piper MD (2007) Optimization of dietary restriction protocols in Drosophila. The journals of gerontology Series A, Biological sciences and medical sciences 62, 1071-81.

34 Piper MD \& Partridge L (2007) Dietary restriction in Drosophila: delayed aging or experimental artefact? PLoS genetics 3, e57.

35 Ziehm M, Piper MD \& Thornton JM (2013) Analysing variation in Drosophila aging across independent experimental studies: a meta-analysis of survival data. Aging cell 12, 917-22.

36 Piper MD (2017) Using artificial diets to understand the nutritional physiology of Drosophila melanogaster. Current opinion in insect science 23.

37 Hosking CJ, Raubenheimer D, Charleston MA, Simpson SJ \& Senior AM (2019) Macronutrient intakes and the lifespan-fecundity trade-off: a geometric framework agent-based model. Journal of The Royal Society Interface 16, 20180733.

38 Moatt JP, Fyfe MA, Heap E, Mitchell LJ, Moon F \& Walling CA (2018) Reconciling nutritional geometry with classical dietary restriction: Effects of nutrient intake, not calories, on survival and reproduction. Aging Cell 18, e12868.

39 Mirth CK, Alves AN \& Piper MD (2018) Turning Food Into Eggs: insights from nutritional biology and developmental physiology of Drosophila. Current Opinion in Insect Science.

40 Wu Q, Yu G, Cheng X, Gao Y, Fan X, Yang D, Xie M, Wang T, Piper MDW \& Yang M (2020)

Sexual dimorphism in the nutritional requirement for adult lifespan in Drosophila melanogaster.

Aging Cell, e13120.

41 Heier C, Knittelfelder O, Hofbauer HF, Mende W, Pörnbacher I, Schiller L, Schoiswohl G, Xie H, Grönke S, Shevchenko A \& Kühnlein RP (2021) Hormone-sensitive lipase couples

intergenerational sterol metabolism to reproductive success. Elife 10, e63252.

42 Zanco B, Rapley L, Johnstone JN, Dedman A, Mirth CK, Sgrò CM \& Piper MD (2021)

Drosophila melanogaster females prioritise dietary sterols for producing high quality eggs. Biorxiv, 2021.06.04.447167.

43 Rera M, Bahadorani S, Cho J, Koehler CL, Ulgherait M, Hur JH, Ansari WS, Lo T, Jones DL \& Walker DW (2011) Modulation of longevity and tissue homeostasis by the Drosophila PGC-1 homolog. Cell metabolism 14, 623-34.

44 Ahmed SMH, Maldera JA, Krunic D, Paiva-Silva GO, Pénalva C, Teleman AA \& Edgar BA (2020) Fitness trade-offs incurred by ovary-to-gut steroid signalling in Drosophila. Nature, 1-5. $45 \mathrm{Li} \mathrm{H} \&$ Jasper H (2016) Gastrointestinal stem cells in health and disease: from flies to humans. Disease Models \& Mechanisms 9, 487-499.

46 Martins R, McCracken A, Simons M, Henriques C \& Rera M (2018) How to Catch a Smurf? Ageing and Beyond... In vivo Assessment of Intestinal Permeability in Multiple Model Organisms. Bio-protocol 8.

47 Regan JC, Lu Y-X, Ureña E, Meilenbrock R, Catterson JH, Kißler D \& Partridge L (2021) Sexual identity of enterocytes regulates rapaymcin-mediated intestinal homeostasis and lifespan extension. bioRxiv.

48 Regan JC, Khericha M, Dobson AJ, Bolukbasi E, Rattanavirotkul N \& Partridge L (2016) Sex difference in pathology of the ageing gut mediates the greater response of female lifespan to dietary restriction. eLife 5, e10956.

49 Bolukbasi E, Khericha M, Regan JC, Ivanov DK, Adcott J, Dyson MC, Nespital T, Thornton JM, Alic N \& Partridge L (2017) Intestinal Fork Head Regulates Nutrient Absorption and Promotes Longevity. Cell Reports 21.

50 Arrese EL \& Soulages JL (2010) Insect Fat Body: Energy, Metabolism, and Regulation. Annu Rev Entomol 55, 207-225.

51 Zipper L, Jassmann D, Burgmer S, Görlich B \& Reiff T (2020) Ecdysone steroid hormone remote controls intestinal stem cell fate decisions via the PPARy-homolog Eip75B in Drosophila. Elife 9, e55795. 
47252 Hayek SA, Alsawadi A, Kambris Z, Boquete J, Bohère J, Immarigeon C, Ronsin B, Plaza S,

473

474

475

476

477

478

479

480

481

482

483

484

485

486

487

488

489

490

491

492

493

494

495

496

497

498

499

500

501

502

503

504

505

506

507

508

509

510

511

512

513

514

515

516

517

518

519

520

521

522

523

524

525

526

527

528

Lemaitre B, Payre F \& Osman D (2020) Steroid-dependent switch of OvoL/Shavenbaby controls self-renewal versus differentiation of intestinal stem cells. Embo J, e104347.

53 Bonfini A, Dobson AJ, Duneau D, Revah J, Liu X, Houtz P \& Buchon N (2021) Multiscale analysis reveals that diet-dependent midgut plasticity emerges from alterations in both stem cell niche coupling and enterocyte size. Elife 10, e64125.

54 Ponton F, Wilson K, Holmes A, Raubenheimer D, Robinson KL \& Simpson SJ (2015)

Macronutrients mediate the functional relationship between Drosophila and Wolbachia.

Proceedings of the Royal Society of London B: Biological Sciences 282, 20142029.

55 Kapahi P, Zid BM, Harper T, Koslover D, Sapin V \& Benzer S (2004) Regulation of Lifespan in Drosophila by Modulation of Genes in the TOR Signaling Pathway. Current Biology 14.

56 Tricoire H, Battisti V, Trannoy S, Lasbleiz C, Pret A-M \& Monnier V (2009) The steroid hormone receptor EcR finely modulates Drosophila lifespan during adulthood in a sex-specific manner.

Mechanisms of Ageing and Development 130, 547552.

57 Knittelfelder O, Prince E, Sales S, Fritzsche E, Wöhner T, Brankatschk M \& Shevchenko A

(2020) Sterols as dietary markers for Drosophila melanogaster. Biochimica Et Biophysica Acta Bba - Mol Cell Biology Lipids 1865, 158683.

58 Markow TA (2015) The secret lives of Drosophila flies. eLife 4.

59 Wilson K \& McLeod BJ (1976) The influence of conditions of growth on the endogenous

metabolism of Saccharomyces cerevisiae: effect on protein, carbohydrate, sterol and fatty acid content and on viability. Antonie Van Leeuwenhoek 42, 397-410.

60 Andreasen AA \& Stier TJB (1953) Anaerobic nutrition of Saccharomyces cerevisiae. I.

Ergosterol requirement for growth in a defined medium. $J$ Cell Compar Phys/ 41, 23-36.

61 Pérez-Torrado R, Gamero E, Gómez-Pastor R, Garre E, Aranda A \& Matallana E (2015) Yeast biomass, an optimised product with myriad applications in the food industry. Trends Food Sci Tech 46, 167-175.

62 Zavrel M, Hoot SJ \& White TC (2013) Comparison of Sterol Import under Aerobic and Anaerobic Conditions in Three Fungal Species, Candida albicans, Candida glabrata, and Saccharomyces cerevisiae. Eukaryot Cell 12, 725-738.

63 Becher PG, Flick G, Rozpędowska E, Schmidt A, Hagman A, Lebreton S, Larsson MC, Hansson BS, Piškur J, Witzgall P \& Bengtsson M (2012) Yeast, not fruit volatiles mediate Drosophila melanogaster attraction, oviposition and development. Funct Ecol 26, 822-828. 64 Walker SJ, Goldschmidt D \& Ribeiro C (2017) Craving for the future: the brain as a nutritional prediction system. Current opinion in insect science 23.

65 Leitão-Gonçalves R, Carvalho-Santos Z, Francisco AP, Fioreze GT, Anjos M, Baltazar C, Elias AP, Itskov PM, Piper MDW \& Ribeiro C (2017) Commensal bacteria and essential amino acids control food choice behavior and reproduction. PLOS Biology 15, e2000862.

66 Warne RW (2014) The Micro and Macro of Nutrients across Biological Scales. Integr Comp Biol 54, 864-872.

67 Carvalho M, Schwudke D, Sampaio JL, Palm W, Riezman I, Dey G, Gupta GD, Mayor S, Riezman H, Shevchenko A, Kurzchalia TV \& Eaton S (2010) Survival strategies of a sterol auxotroph. Development 137, 3675-85.

68 ROSEWELL J \& SHORROCKS B (1987) The implication of survival rates in natural populations of Drosophila: capture-recapture experiments on domestic species. Biol J Linn Soc 32, 373-384. 69 Kawasaki N, Brassil CE, Brooks RC \& Bonduriansky R (2008) Environmental Effects on the Expression of Life Span and Aging: An Extreme Contrast between Wild and Captive Cohorts ofTelostylinus angusticollis(Diptera: Neriidae). The American Naturalist 172, 346-357.

70 Bonduriansky R \& Brassil CE (2002) Rapid and costly ageing in wild male flies. Nature 420, 377-377.

71 Ja WW, Carvalho GB, Zid BM, Mak EM, Brummel T \& Benzer S (2009) Water- and nutrientdependent effects of dietary restriction on Drosophila lifespan. Proceedings of the National Academy of Sciences 106, 18633-18637.

72 McCracken AW, Buckle E \& Simons MJP (2020) The relationship between longevity and diet is genotype dependent and sensitive to desiccation in Drosophila melanogaster. J Exp Biol, jeb.230185.

73 Piper, Wong R, Grandison R, Bass T, Martinez P \& Partridge L (2010) Water-independent effects of dietary restriction in Drosophila. Proceedings of the National Academy of Sciences of the 
529 United States of America 107, E54-6; author reply E57.

53074 Piper MD \& Partridge L (2016) Protocols to Study Aging in Drosophila. Methods in molecular 531 biology (Clifton, NJ) 1478, 291-302.

532

533

534

535

536

537

538

539

540

541

542

543

544

545

546

547

548

549

550

551

552

553

554

555

556

557

558

559

560

561

562

563

564

565

566

567

75 Lee KP, Simpson SJ, Clissold FJ, Brooks R, Ballard JW, Taylor PW, Soran N \& Raubenheimer D (2008) Lifespan and reproduction in Drosophila: New insights from nutritional geometry.

Proceedings of the National Academy of Sciences of the United States of America 105, 2498-503. 76 Skorupa DA, Dervisefendic A, Zwiener J \& Pletcher SD (2008) Dietary composition specifies consumption, obesity, and lifespan in Drosophila melanogaster. Aging Cell 7, 478-490.

77 Chandegra B, Tang JLYL, Chi H \& Alic N (2017) Sexually dimorphic effects of dietary sugar on lifespan, feeding and starvation resistance in Drosophila. Aging 9, 2521-2528.

78 Simpson SJ \& Raubenheimer D (1993) A multi-level analysis of feeding behaviour: the geometry of nutritional decisions. Philosophical Transactions Royal Soc Lond Ser B Biological Sci 342, 381-402.

79 Sperfeld E, Raubenheimer D \& Wacker A (2016) Bridging factorial and gradient concepts of resource co-limitation: towards a general framework applied to consumers. Ecol Lett 19, 201-215. 80 Wacker A \& Martin-Creuzburg D (2012) Biochemical nutrient requirements of the rotifer Brachionus calyciflorus: co-limitation by sterols and amino acids. Funct Ecol 26, 1135-1143. 81 Sperfeld E, Martin-Creuzburg D \& Wacker A (2012) Multiple resource limitation theory applied to herbivorous consumers: Liebig's minimum rule vs. interactive co-limitation. Ecol Lett 15, $142-$ 150.

82 Martin-Creuzburg D, Sperfeld E \& Wacker A (2009) Colimitation of a freshwater herbivore by sterols and polyunsaturated fatty acids. Proc Royal Soc B Biological Sci 276, 1805-1814.

83 Speakman JR (2008) The physiological costs of reproduction in small mammals. Philosophical Transactions Royal Soc B Biological Sci 363, 375-398.

84 Miller SC \& Bowman BM (2004) Rapid improvements in cortical bone dynamics and structure after lactation in established breeder rats. Anatomical Rec Part Discov Mol Cell Evol Biology 276A, 143-149.

85 Speakman JR, Mitchell SE \& Mazidi M (2016) Calories or protein? The effect of dietary restriction on lifespan in rodents is explained by calories alone. Experimental Gerontology 86, 2838.

86 Solon-Biet SM, McMahon AC, Ballard WOJ, Ruohonen K, Wu LE, Cogger VC, Warren A, Huang X, Pichaud N, Melvin RG, Gokarn R, Khalil M, Turner N, Cooney GJ, Sinclair DA, Raubenheimer D, Le Couteur DG \& Simpson SJ (2014) The Ratio of Macronutrients, Not Caloric Intake, Dictates Cardiometabolic Health, Aging, and Longevity in Ad Libitum-Fed Mice. Cell Metabolism 19.

87 Promislow DEL, Flatt T \& Bonduriansky R (2021) The Biology of Aging in Insects: From Drosophila to Other Insects and Back. Annu Rev Entomol 67, 1-21.

88 Zajitschek F, Zajitschek S \& Bonduriansky R (2020) Senescence in wild insects: Key questions and challenges. Funct Ecol 34, 26-37. 